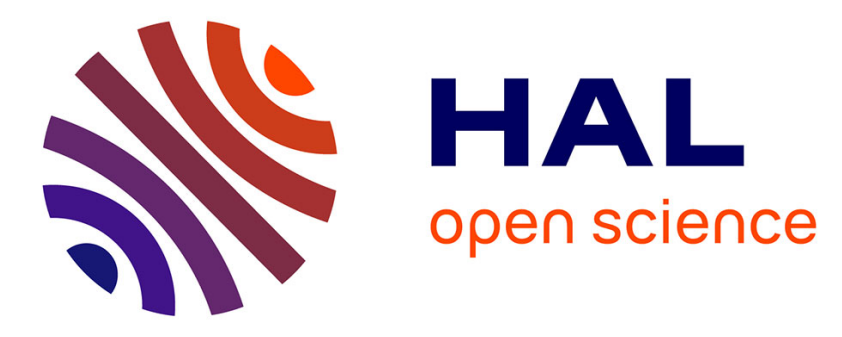

\title{
Effect of quenching rate on SDW dynamics in (TMTSF)2ClO4
}

K. Nomura, T. Onodera, T. Shimizu, T. Sambongi, M. Tokumoto, N.

Kinoshita, H. Anzai

\section{- To cite this version:}

K. Nomura, T. Onodera, T. Shimizu, T. Sambongi, M. Tokumoto, et al.. Effect of quenching rate on SDW dynamics in (TMTSF)2ClO4. Journal de Physique IV Proceedings, 1993, 03 (C2), pp.C2-263C2-266. 10.1051/jp4:1993253 . jpa-00251336

\section{HAL Id: jpa-00251336 https://hal.science/jpa-00251336}

Submitted on 1 Jan 1993

HAL is a multi-disciplinary open access archive for the deposit and dissemination of scientific research documents, whether they are published or not. The documents may come from teaching and research institutions in France or abroad, or from public or private research centers.
L'archive ouverte pluridisciplinaire HAL, est destinée au dépôt et à la diffusion de documents scientifiques de niveau recherche, publiés ou non, émanant des établissements d'enseignement et de recherche français ou étrangers, des laboratoires publics ou privés. 


\title{
Effect of quenching rate on SDW dynamics in (TMTSF) ${ }_{2} \mathrm{ClO}_{4}$
}

\author{
K. NOMURA, T. ONODERA, T. SHIMIZU, T. SAMBONGI, M. TOKUMOTO*, N. KINOSHITA* \\ and $\mathrm{H}$. ANZAI ${ }^{* *}$
}

Department of Physics, Hokkaido University, Sapporo 060, Japan

${ }^{*}$ Electrotechnical Laboratory, Tsukuba, Ibaraki 305, Japan

** Department of Material Science, Himeji Institute of Technology, Kamigohri, Hyogo 678-12, Japan

\begin{abstract}
The pinning mechanism of SDW condensate associated with the imperfect nesting effect was investigated with the measurement of the temperature dependence of the threshold electric field $\mathrm{E}_{\mathrm{T}}$ for the depinning of SDW in (TMTSF) ${ }_{2} \mathrm{ClO}_{4}$ for various anion quenching rates. The relatively strong temperature dependence of $\mathrm{E}_{\mathrm{T}}$ observed in the most rapidly quenched phase with $T_{C}=5.85 \mathrm{~K}$ is described by the $3-$ dimensional impurity weak pinning. With decreasing the quenching rate, the temperature dependence of $\mathrm{E}_{\mathrm{T}}$ becomes gradual for $\mathrm{T}_{\mathrm{C}}>3 \mathrm{~K}$. This behavior is explained by the change of pinning mechanism to the strong pinning. For much lower quenching rate $\left(\mathrm{T}_{\mathrm{C}}<3 \mathrm{~K}\right)$, the temperature dependence of $\mathrm{E}_{\mathrm{T}}$ becomes steeper again and is attributed to the increase of imperfectness of nesting of Fermi surface. It is understood that the SDW pinning is provided by the disordered array of anion.
\end{abstract}

\section{INTRODUCTION}

The collective sliding mode of spin-density wave (SDW) condensate have been investigated extensively in the SDW phase of (TMTSF) ${ }_{2} X$ salts. The early observation of the non-linear conductivity was explained rather by the one-electron effect than the collective mode, as the threshold electric field was absent unlike the case of the charge-density wave. Tomic et al. [1] reported the non-linear conductivity with a clear threshold field for the depinning of SDW in (TMTSF) ${ }_{2} \mathrm{NO}_{3}$. Similar threshold behavior was also found in quenched (TMTSF) ${ }_{2} \mathrm{ClO}_{4}$ [2] and (TMTSF) ${ }_{2} \mathrm{PF}_{6}$ [3] in succession. The narrow band voltage noise was successfully measured in the SDW phase of (TMTSF) ${ }_{2} \mathrm{ClO}_{4}$ [4]. The narrowing of the NMR line structure associated with the SDW by its sliding motion was also observed in (TMTSF) ${ }_{2} \mathrm{ClO}_{4}$ [5]. The sliding mode of SDW was established by these experiments unambiguously.

It is important to clarify the pinning mechanism for better understanding of the dynamics of SDW. The pinning of SDW by non-magnetic impurities is of second order in contrast with the case of the charge-density wave, because the charge density is uniform in space for the SDW state. Huang et al. [6] calculated the threshold electric field $E_{T}$ for the impurity pinning within the mean field treatment and tried to explain observed temperature dependence of $\mathrm{E}_{\mathrm{T}}$ in several (TMTSF) ${ }_{2} \mathrm{X}$ salts. The temperature dependence of the order parameter, which is determined by the two-dimensionality of initial electronic band, plays an essential role in their theory. With increasing the two-dimensional character in the electronic band, the nesting of Fermi surface becomes less perfect. It is expected that with increasing the imperfectness of nesting, the temperature dependence of $E_{T}$ becomes steeper for both the strong and the weak impurity pinning.

In (TMTSF) ${ }_{2} \mathrm{ClO}_{4}$ salt, there is the orientational order transition of non-centrosymmetric anion $\mathrm{ClO}_{4}^{-}$around $24 \mathrm{~K}$. The degree of anion ordering determines the ground state through the twodimensionality in electronic band. When the sample is cooled down slowly through this temperature, the superconducting phase appears. On the other hand, in the rapidly quenched phase the SDW state is stabilized as the ground state. It was reported that with decreasing the quenching rate, the SDW transition temperature $T_{C}$ decreases. It is accepted that such a decreasing of $T_{C}$ is provided by the 
decreasing of perfectness of Fermi surface nesting. Accordingly (TMTSF) ${ }_{2} \mathrm{ClO}_{4}$ is a suitable materials for investigation of the imperfect nesting effect.

In order to clarify the imperfect nesting effect to the SDW pinning, we investigated the temperature dependence of threshold electric field $\mathrm{E}_{\mathrm{T}}$ with varying the quenching rate systematically in (TMTSF $)_{2} \mathrm{ClO}_{4}$. In this article, we report the observed behavior of $\mathrm{E}_{\mathrm{T}}$ and discuss the pinning mechanism by the disordered array of anion.

\section{EXPERIMENTAL}

Crystal of (TMTSF) ${ }_{2} \mathrm{ClO}_{4}$ was grown by the electro-chemical method. We investigated two sample from different batches; \#1 and \#2. The electric conductivity was measured mainly by the pulse method with standard four-probe in order to avoid Joule heating effect. Electric lead of $10 \mu \mathrm{m}$ gold wire was attached to gold evaporated pads with silver paint. Typical sample cross-section and distance between voltage leads were $0.2 \times 0.1 \times 5 \mathrm{~mm}^{3}$. In the initial cooling stage down to $77 \mathrm{~K}$, the cooling rate was controlled less than $4 \mathrm{~K} / \mathrm{h}$ to avoid the occurrence of resistance jump. The quenching was done by immersing the sample into liquid helium directly after pre-holding at $40 \mathrm{~K}$. The quenching rate was defined as the rate of decreasing temperature through the anion ordering temperature $24 \mathrm{~K}$. This rate was monitored by the silicon-diode thermometer. The SDW transition temperature was determined from the peak of $d(\operatorname{lnR}) / d(1 / T)$, where $R$ is the ohmic resistance.

\section{RESULTS AND DISCUSSION}

The quenching rate was controlled manually in the empirically determined manner. The observed SDW transition temperature $T_{C}$ is shown as a function of the quenching rate in Fig. 1. For the quenching rate higher than $50 \mathrm{~K} / \mathrm{h}, \mathrm{T}_{\mathrm{C}}$ is almost unchanged. With decreasing the quenching rate below $50 \mathrm{~K} / \mathrm{h}, \mathrm{T}_{\mathrm{C}}$ begins to decrease as shown in Fig. 1 . We confirmed that $\mathrm{T}_{\mathrm{C}}$ is systematically controlled by the quenching rate in our present method. This decreasing of $T_{C}$ is explained by the increase of the imperfectness parameter $\varepsilon_{0}$ defined as

$$
\varepsilon_{0}=\mathrm{t}_{\mathrm{b}}{ }^{2} \cos \left(\mathrm{ak}_{\mathrm{F}}\right) / 2 \mathrm{t}_{\mathrm{a}} \sin ^{2}\left(\mathrm{ak}_{\mathrm{F}}\right)
$$

where $t_{a}$ and $t_{b}$ are transfer energies along the $a-$ and $b$-axis, respectively. The ratio of $\varepsilon_{0}$ to the order parameter at $0 \mathrm{~K} \Delta$ is a fundamental parameter and the SDW phase disappears for $\varepsilon_{0} / \Delta>1$. It is accordingly understood that with increasing the anion ordering the nesting becomes less perfect.
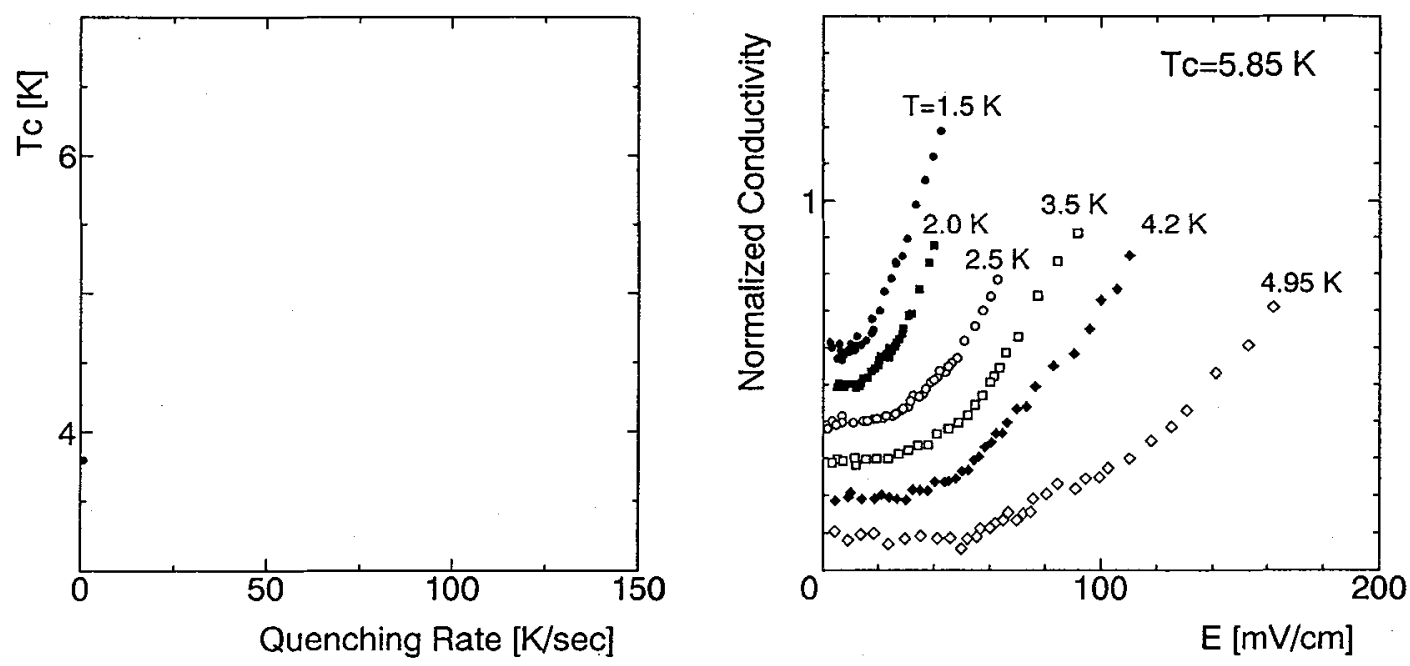

Fig. 1 SDW transition temperature vs. anion quenching rate.

Fig. 2 Normalized excess conductivity vs. electric field for the rapidly quenched phase in \#1 sample. 
In Fig. 2, we show the field dependent normalized conductivity $\{\sigma(E)-\sigma(0)\} / \sigma(0)$ in the rapidly quenched SDW phase with $\mathrm{T}_{\mathrm{C}}=5.85 \mathrm{~K}$ measured in \#1 sample for several temperatures. Each curve is offset by 0.1 for clarity. The conductivity is increasing above the well-defined threshold electric field $E_{\mathrm{T}}$. We determined $E_{\mathrm{T}}$ as the intersection of the ohmic conductivity line and the extrapolated line from $2 \mathrm{E}_{\mathrm{T}}$ as described in the previous article [7].

The temperature dependence of $E_{T}$ in $\# 1$ sample is shown for each quenching rate in Fig. 3 . In the figure, the quenching rate is denoted as the transition temperature $T_{C}$. For the most rapid quenching with $\mathrm{T}_{C}=5.85 \mathrm{~K}$, the temperature dependence of $\mathrm{E}_{\mathrm{T}}$ is relatively steep and is consistent with the previous result [7]. This temperature dependence is well fitted by the theoretical curve of 3dimensional weak impurity pinning with imperfectness parameter $\varepsilon_{0} / \Delta=0.7$, where the order parameter is deformable in 3-dimensional space. With lowering $\mathrm{T}_{\mathrm{C}}$, the temperature dependence of $\mathrm{E}_{\mathrm{T}}$ becomes gradual. It is expected from the mean field treatment that the temperature dependence of $\mathrm{E}_{\mathrm{T}}$ become steeper along with the increase of imperfectness of nesting in the $3 \mathrm{~d}$ weak pinning. The observed curve shows an opposite behavior and is fitted rather by the strong pinning with $\varepsilon_{0} / \Delta=0.8$ than the weak one as shown in Fig. 3. The strong pinning occurs when the individual impurity potential is strong and the impurity concentration is small. In quenched (TMTSF) ${ }_{2} \mathrm{ClO}_{4}$, the impurity potential is assigned to the disordered array of anion, which breaks the translational symmetry. It is naturally expected that the disorder density is smaller for a phase with lower $T_{c}$. It is understand the weak pinning changes to the strong one, with decreasing disorder.
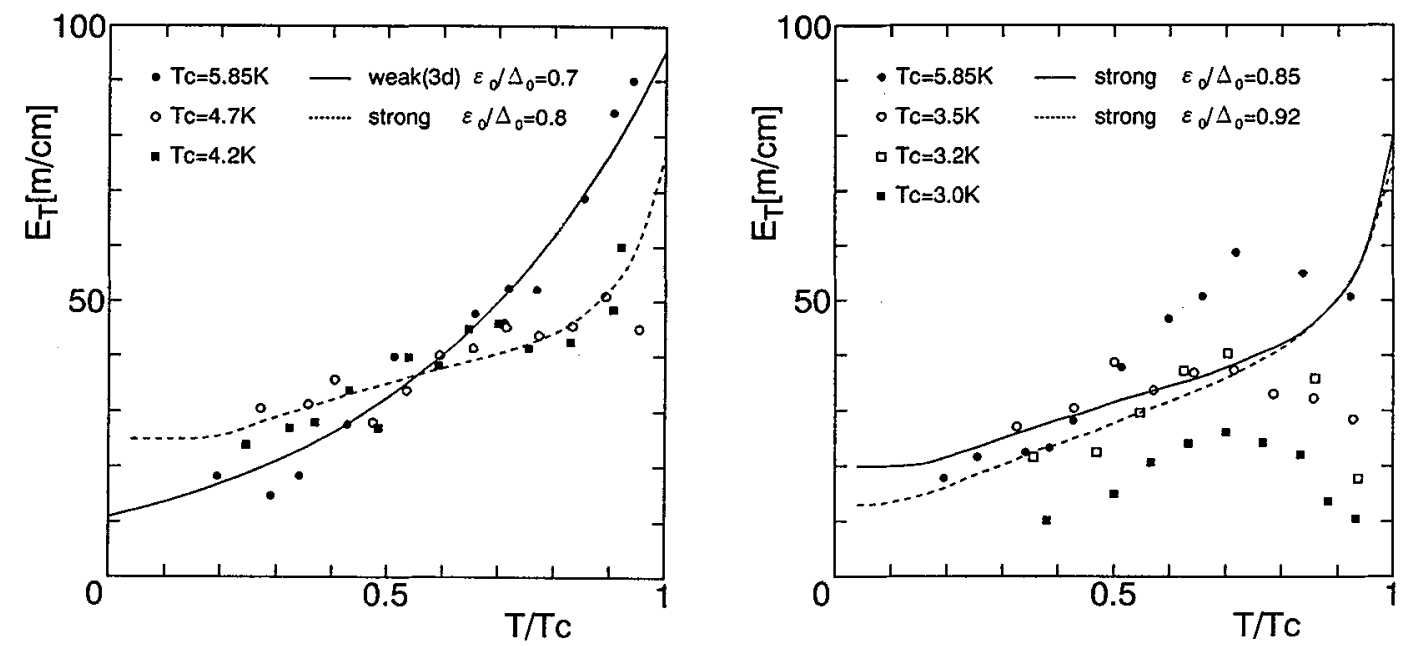

Fig. 3 Temperature dependence of $E_{\mathrm{T}}$ in $\# 1$ sample for various quenching rates and fitting to the theoretical calculation. The quenching rate is denoted by $\mathrm{T}_{\mathrm{C}}$.

Fig. 4 Temperature dependence of $\mathrm{E}_{\mathrm{T}}$ in $\# 2$ sample for various quenching rates and fitting to the theoretical calculation.

Further slow quenching phase was examined in \#2 sample. The temperature dependence of $E_{T}$ is shown in Fig. 4. In this sample, $\mathrm{E}_{\mathrm{T}}$ shows an anomalous decrease at high temperature region. We observed that the fractional excess conductivity is decreasing abruptly in this anomalous temperature region. It is deduced that the SDW is sliding in only limited volume of sample at high temperature region. Some inhomogeneity in the sample may be responsible for such an anomalous behavior. On the other hand, the behavior at low temperature region is essentially the same as \#1 sample and other samples investigated in the previous work. Accordingly, we discuss here only the result in the low temperature for \#2 sample. We observed essentially the same temperature dependence of $E_{T}$ as \#1 sample for higher $\mathrm{T}_{\mathrm{C}}$ than $3.5 \mathrm{~K}$, although only the result for $\mathrm{T}_{\mathrm{C}}=5.85 \mathrm{~K}$ is shown in Fig. 4 for clarity. With lowering $\mathrm{T}_{\mathrm{c}}$ below $3.5 \mathrm{~K}$, the temperature dependence of $\mathrm{E}_{\mathrm{T}}$ becomes steeper again. The absolute magnitude of $E_{T}$ at low reduced temperature $\left(T / T_{c}=0.3\right)$ decreases with lowering $T_{c}$. Within the strong pinning regime, the temperature dependence of $\mathrm{E}_{\mathrm{T}}$ is dominated by that of order parameter $\Delta(\mathrm{T})$. As shown in Fig. 4, the observed temperature dependence is well reproduced by the theoretical 
calculation taking account of the imperfectness parameter.

Table 1 Imperfectness parameter $\varepsilon_{0} / \Delta$ estimated from $T_{C}$ and the temperature dependence of $E_{T}$

\begin{tabular}{c|cc}
\hline \multirow{2}{*}{$\mathrm{T}_{\mathrm{C}}(\mathrm{K})$} & \multicolumn{2}{|c}{$\varepsilon_{0} / \Delta$} \\
\cline { 2 - 3 } & from $\mathrm{T}_{\mathrm{C}}$ & from $\mathrm{E}_{\mathrm{T}}$ \\
\hline 5.85 & 0.7 & 0.7 \\
4.2 & 0.9 & 0.8 \\
3.5 & 0.95 & 0.85 \\
3.2 & 0.96 & 0.92 \\
\hline
\end{tabular}

In table 1 , we give estimated values for the imperfectness parameter $\varepsilon_{0} / \Delta$ from the relation between $T_{C}$ and $\varepsilon_{0} / \Delta$ in the mean field theory together with that used in the fitting of observed temperature dependent $E_{T}$. The agreement is satisfactorily and it strongly supports the above mentioned mechanism. The absolute magnitude of $E_{T}$ decreasing with $T_{c}$ is also reasonably explained by the strong pinning where $E_{T}$ is proportional to the impurity concentration, because the disorder density is expected to be smaller for lower $\mathrm{T}_{\mathrm{C}}$. The SDW pinning in quenched (TMTSF) ${ }_{2} \mathrm{ClO}_{4}$ is totally understood as the impurity pinning by the disordered array of anion in connection with the imperfect nesting effect.

\section{REFERENCES}

1. S. Tomić, J. R. Cooper, D. Jérome and K. Bechgaard, Phys. Rev. Lett., 62(1989)462.

2. T. Sambongi, K. Nomura, T. Shimizu, K. Ichimura, N. Kinoshita, M. Tokumoto and H. Anzai, Solid State Commun., 72(1989)817.

3. W. Kang, S. Tomić, J. R. Cooper and D. Jérome, Phys. Rev. B41(1990)4862.

4. K. Nomura, T. Shimizu, K. Ichimura, T. Sambongi, M. Tokumoto, H. Anzai and N. Kinoshita, Solid State Commun., 72(1989)1123.

5. M. J. Delrieu and N. Kinoshita, Compt Rendus, 310(1990)891.

6. X. Huang and K. Maki, Phys. Rev., B42(1990)6498.

7. T. Shimizu, K. Nomura, T. Sambongi, H. Anzai, N. Kinoshita and M. Tokumoto, Solid State Commun., 78(1991)697. 